\title{
ToTTo: A Controlled Table-To-Text Generation Dataset
}

\author{
Ankur P. Parikh ${ }^{\star} \quad$ Xuezhi Wang ${ }^{\star}$ Sebastian Gehrmann ${ }^{\star}$ \\ Manaal Faruqui $^{\star}$ Bhuwan Dhingra** Diyi Yang $\diamond$ Dipanjan Das ${ }^{\star}$ \\ - Google Research, New York, NY \\ $\diamond$ Georgia Tech, Atlanta, GA \\ * Carnegie Mellon University, Pittsburgh, PA \\ totto@google.com
}

\begin{abstract}
We present TOTTO, an open-domain English table-to-text dataset with over 120,000 training examples that proposes a controlled generation task: given a Wikipedia table and a set of highlighted table cells, produce a one-sentence description. To obtain generated targets that are natural but also faithful to the source table, we introduce a dataset construction process where annotators directly revise existing candidate sentences from Wikipedia. We present systematic analyses of our dataset and annotation process as well as results achieved by several state-of-the-art baselines. While usually fluent, existing methods often hallucinate phrases that are not supported by the table, suggesting that this dataset can serve as a useful research benchmark for high-precision conditional text generation. ${ }^{1}$
\end{abstract}

\section{Introduction}

Data-to-text generation (Kukich, 1983; McKeown, 1992) is the task of generating a target textual description $\boldsymbol{y}$ conditioned on source content $\boldsymbol{x}$ in the form of structured data such as a table. Examples include generating sentences given biographical data (Lebret et al., 2016), textual descriptions of restaurants given meaning representations (Novikova et al., 2017), basketball game summaries given boxscore statistics (Wiseman et al., 2017), and generating fun facts from superlative tables in Wikipedia (Korn et al., 2019).

Existing data-to-text tasks have provided an important test-bed for neural generation models (Sutskever et al., 2014; Bahdanau et al., 2014). Neural models are known to be prone to hallucination, i.e., generating text that is fluent but not faithful to the source (Vinyals and Le, 2015; Koehn

\footnotetext{
${ }^{*}$ Work done during an internship at Google.

${ }^{1}$ TOTTO is available at https://github.com/ google-research-datasets/totto.
}

and Knowles, 2017; Lee et al., 2018; Tian et al., 2019) and it is often easier to assess faithfulness of the generated text when the source content is structured (Wiseman et al., 2017; Dhingra et al., 2019). Moreover, structured data can also test a model's ability for reasoning and numerical inference (Wiseman et al., 2017) and for building representations of structured objects (Liu et al., 2018), providing an interesting complement to tasks that test these aspects in the NLU setting (Pasupat and Liang, 2015; Chen et al., 2019; Dua et al., 2019).

However, constructing a data-to-text dataset can be challenging on two axes: task design and annotation process. First, tasks with open-ended output like summarization (Mani, 1999; Lebret et al., 2016; Wiseman et al., 2017) lack explicit signals for models on what to generate, which can lead to subjective content and evaluation challenges (Kryściński et al., 2019). On the other hand, data-to-text tasks that are limited to verbalizing a fully specified meaning representation (Gardent et al., 2017b) do not test a model's ability to perform inference and thus remove a considerable amount of challenge from the task.

Secondly, designing an annotation process to obtain natural but also clean targets is a significant challenge. One strategy employed by many datasets is to have annotators write targets from scratch (Banik et al., 2013; Wen et al., 2015; Gardent et al., 2017a) which can often lack variety in terms of structure and style (Gururangan et al., 2018; Poliak et al., 2018). An alternative is to pair naturally occurring text with tables (Lebret et al., 2016; Wiseman et al., 2017). While more diverse, naturally occurring targets are often noisy and contain information that cannot be inferred from the source. This can make it problematic to disentangle modeling weaknesses from data noise.

In this work, we propose TOTTO, an opendomain table-to-text generation dataset that intro- 
Table Title: Gabriele Becker

Section Title: International Competitions

Table Description: None

\begin{tabular}{|c|c|c|c|c|c|}
\hline Year & Competition & Venue & Position & Event & Notes \\
\hline \multicolumn{6}{|c|}{ Representing Germany } \\
\hline 1992 & World Junior Championships & Seoul, South Korea & 10th (semis) & $100 \mathrm{~m}$ & 11.83 \\
\hline \multirow{2}{*}{1993} & \multirow{2}{*}{ European Junior Championships } & \multirow{2}{*}{ San Sebastián, Spain } & 7th & $100 \mathrm{~m}$ & 11.74 \\
\hline & & & 3rd & $4 \times 100$ m relay & 44.60 \\
\hline \multirow{2}{*}{1994} & \multirow{2}{*}{ World Junior Championships } & \multirow{2}{*}{ Lisbon, Portugal } & 12th (semis) & $100 \mathrm{~m}$ & 11.66 (wind: $+1.3 \mathrm{~m} / \mathrm{s}$ ) \\
\hline & & & 2nd & $4 \times 100$ m relay & 44.78 \\
\hline \multirow{2}{*}{1995} & \multirow{2}{*}{ World Championships } & \multirow{2}{*}{ Gothenburg, Sweden } & 7th (q-finals) & \multirow{2}{*}{$\begin{array}{c}100 \mathrm{~m} \\
4 \times 100 \mathrm{~m} \text { relay }\end{array}$} & 11.54 \\
\hline & & & 3rd & & 43.01 \\
\hline
\end{tabular}

Original Text: After winning the German under-23 $100 \mathrm{~m}$ title, she was selected to run at the 1995 World Championships in Athletics both individually and in the relay.

Text after Deletion: she at the 1995 World Championships in both individually and in the relay.

Text After Decontextualization: Gabriele Becker competed at the 1995 World Championships

in both individually and in the relay.

Final Text: Gabriele Becker competed at the 1995 World Championships both individually and in the relay.

Table 1: Example in the ToTTo dataset. The goal of the task is given the table, table metadata (such as the title), and set of highlighted cells, to produce the final text. Our data annotation process revolves around annotators iteratively revising the original text to produce the final text.

duces a novel task design and annotation process to address the above challenges. First, TOTTO proposes a controlled generation task: given a Wikipedia table and a set of highlighted cells as the source $\boldsymbol{x}$, the goal is to produce a single sentence description $\boldsymbol{y}$. The highlighted cells identify portions of potentially large tables that the target sentence should describe, without specifying an explicit meaning representation to verbalize.

For dataset construction, to ensure that targets are natural but also faithful to the source table, we request annotators to revise existing Wikipedia candidate sentences into target sentences, instead of asking them to write new target sentences (Wen et al., 2015; Gardent et al., 2017a). Table 1 presents a simple example from TOTTo to illustrate our annotation process. The table and Original Text were obtained from Wikipedia using heuristics that collect pairs of tables $\boldsymbol{x}$ and sentences $\boldsymbol{y}$ that likely have significant semantic overlap. This method ensures that the target sentences are natural, although they may only be partially related to the table. Next, we create a clean and controlled generation task by requesting annotators to highlight a subset of the table that supports the original sentence and revise the latter iteratively to produce a final sentence (see $\S 5)$. For instance, in Table 1, the annotator has chosen to highlight a set of table cells (in yellow) that support the original text. They then deleted phrases from the original text that are not supported by the table, e.g., After winning the German under-23 100 $m$ title, and replaced the pronoun she with an entity
Gabriele Becker. The resulting final sentence ( $\mathrm{Fi}$ nal Text) serves as a more suitable generation target than the original sentence. This annotation process makes our dataset well suited for high-precision conditional text generation.

Due to the varied nature of Wikipedia tables, TOTTO covers a significant variety of domains while containing targets that are completely faithful to the source (see Table 4 and the Appendix for more complex examples). Our experiments demonstrate that state-of-the-art neural models struggle to generate faithful results, despite the high quality of the training data. These results suggest that our dataset could serve as a useful benchmark for controllable data-to-text generation.

\section{Related Work}

TOTTO differs from existing datasets in both task design and annotation process as we describe below. A summary is given in Table 2.

Task Design Most existing table-to-text datasets are restricted in topic and schema such as WEATHERGov (Liang et al., 2009), RовоCup (Chen and Mooney, 2008), Rotowire (Wiseman et al., 2017, basketball), E2E (Novikova et al., 2016, 2017, restaurants), KBGen (Banik et al., 2013, biology), and Wikibio (Lebret et al., 2016, biographies). In contrast, TOTTO contains tables with various schema spanning various topical categories all over Wikipedia. Moreover, TOTTO takes a different view of content selection compared to 


\begin{tabular}{|c|c|c|c|c|c|}
\hline Dataset & Train Size & Domain & Target Quality & Target Source & Content Selection \\
\hline Wikibio (Lebret et al., 2016) & $583 \mathrm{~K}$ & Biographies & Noisy & Wikipedia & Not specified \\
\hline WebNLG (Gardent et al., 2017b) & $25.3 \mathrm{~K}$ & 15 DBPedia categories & Clean & Annotator Generated & Fully specified \\
\hline ToTTo & $120 \mathrm{~K}$ & Wikipedia (open-domain) & Clean & Wikipedia (Annotator Revised) & Annotator highlighted \\
\hline
\end{tabular}

Table 2: Comparison of popular data-to-text datasets. TOTTO combines the advantages of annotator-generated and fully natural text through a revision process.

existing datasets. Prior to the advent of neural approaches, generation systems typically separated content selection (what to say) from surface realization (how to say it) (Reiter and Dale, 1997). Thus many generation datasets only focused on the latter stage (Wen et al., 2015; Gardent et al., 2017b). However, this decreases the task complexity, since neural systems have already been quite powerful at producing fluent text. Some recent datasets (Wiseman et al., 2017; Lebret et al., 2016) have proposed incorporating content selection into the task by framing it as a summarization problem. However, summarization is much more subjective, which can make the task underconstrained and difficult to evaluate (Kryściński et al., 2019). We place TOTTO as a middle-ground where the highlighted cells provide some guidance on the topic of the target but still leave a considerable amount of content planning to be done by the model.

Annotation Process There are various existing strategies to create the reference target $\boldsymbol{y}$. One strategy employed by many datasets is to have annotators write targets from scratch given a representation of the source (Banik et al., 2013; Wen et al., 2015; Gardent et al., 2017a). While this will result in a target that is faithful to the source data, it often lacks variety in terms of structure and style (Gururangan et al., 2018; Poliak et al., 2018). Domainspecific strategies such as presenting an annotator an image instead of the raw data (Novikova et al., 2016) are not practical for some of the complex tables that we consider. Other datasets have taken the opposite approach: finding real sentences on the web that are heuristically selected in a way that they discuss the source content (Lebret et al., 2016; Wiseman et al., 2017). This strategy typically leads to targets that are natural and diverse, but they may be noisy and contain information that cannot be inferred from the source (Dhingra et al., 2019).To construct TOTTO, we ask annotators to revise existing candidate sentences from Wikipedia so that they only contain information that is supported by the table. This enables ToTTo to maintain the varied language and structure found in natural sentences while producing cleaner targets. The technique of editing exemplar sentences has been used in semiparametric generation models (Guu et al., 2018; Pandey et al., 2018; Peng et al., 2019) and crowd-sourcing small, iterative changes to text has been shown to lead to higher-quality data and a more robust annotation process (Little et al., 2010). Perez-Beltrachini and Lapata (2018) also employed a revision strategy to construct a cleaner evaluation set for Wikibio (Lebret et al., 2016).

Concurrent to this work, Chen et al. (2020) proposed LogicNLG which also uses Wikipedia tables, although omitting some of the more complex structured ones included in our dataset. Their target sentences are annotator-generated and their task is significantly more uncontrolled due to the lack of annotator highlighted cells.

\section{Preliminaries}

Our tables come from English Wikipedia articles and thus may not be regular grids. ${ }^{2}$ For simplicity, we define a table $\boldsymbol{t}$ as a set of cells $\boldsymbol{t}=\left\{\boldsymbol{c}_{j}\right\}_{j=1}^{\tau}$ where $\tau$ is the number of cells in the table. Each cell contains: (1) a string value, (2) whether or not it is a row or column header, (3) the row and column position of this cell in the table, (4) The number of rows and columns this cell spans.

Let $\boldsymbol{m}=\left(m_{\text {page-title }}, m_{\text {section-title }}, m_{\text {section-text }}\right)$ indicate table metadata, i.e, the page title, section title, and up to the first 2 sentences of the section text (if present) respectively. These fields can help provide context to the table's contents. Let $s=\left(s_{1}, \ldots, s_{\eta}\right)$ be a sentence of length $\eta$. We define an annotation example ${ }^{3} \boldsymbol{d}=(\boldsymbol{t}, \boldsymbol{m}, \boldsymbol{s})$ a tuple of table, table metadata, and sentence. Here, $\boldsymbol{D}=\left\{\boldsymbol{d}_{n}\right\}_{n=1}^{N}$ refers to a dataset of annotation

\footnotetext{
${ }^{2}$ In Wikipedia, some cells may span multiple rows and columns. See Table 1 for an example.

${ }^{3}$ An annotation example is different than a task example since the annotator could perform a different task than the model.
} 
examples of size $N$.

\section{Dataset Collection}

We first describe how to obtain annotation examples $\boldsymbol{d}$ for subsequent annotation. To prevent any overlap with the Wikibio dataset (Lebret et al., 2016), we do not use infobox tables. We employed three heuristics to collect tables and sentences:

Number matching We search for tables and sentences on the same Wikipedia page that overlap with a non-date number of at least 3 non-zero digits. This approach captures most of the table-sentence pairs that describe statistics (e.g., sports, election, census, science, weather).

Cell matching We extract a sentence if it has tokens matching at least 3 distinct cell contents from the same row in the table. The intuition is that most tables are structured, and a row is usually used to describe a complete event.

Hyperlinks The above heuristics only consider sentences and tables on the same page. We also find examples where a sentence $s$ contains a hyperlink to a page with a title that starts with List (these pages typically only consist of a large table). If the table $t$ on that page also has a hyperlink to the page containing $s$, then we consider this to be an annotation example. Such examples typically result in more diverse examples than the other two heuristics, but also add more noise, since the sentence may only be distantly related to the table.

Using the above heuristics we obtain a set of examples $\boldsymbol{D}$. We then sample a random subset of tables for annotation, excluding tables with formatting issues: 191,693 examples for training, 11,406 examples for development, and 11,406 examples for test. Among these examples, 35.8\% were derived from number matching, $29.4 \%$ from cell matching, and $34.7 \%$ from hyperlinks.

\section{Data Annotation Process}

The collected annotation examples are noisy since a sentence $s$ may only be partially supported by the table $\boldsymbol{t}$. We thus define an annotation process that guides annotators through incremental changes to the original sentence. This allows us to measure annotator agreement at every step of the process, which is atypical in existing generation datasets.

The primary annotation task consists of the following steps: (1) Table Readability, (2) Cell high- lighting, (3) Phrase Deletion, (4) Decontextualization. After these steps we employ a final secondary annotation task for grammar correction. Each of these are described below and more examples are provided in the Table 3.

Table Readability If a table is not readable, then the following steps will not need to be completed. This step is only intended to remove fringe cases where the table is poorly formatted or otherwise not understandable (e.g., in a different language). $99.5 \%$ of tables are determined to be readable.

Cell Highlighting An annotator is instructed to highlight cells that support the sentence. A phrase is supported by the table if it is either directly stated in the cell contents or meta-data, or can be logically inferred by them. Row and column headers do not need to be highlighted. If the table does not support any part of the sentence, then no cell is marked and no other step needs to be completed. $69.7 \%$ of examples are supported by the table. For instance, in Table 1, the annotator highlighted cells that support the phrases 1995, World Championships, individually, and relay. The set of highlighted cells are denoted as a subset of the table: $\boldsymbol{t}_{\text {highlight }} \in \boldsymbol{t}$.

Phrase Deletion This step removes phrases in the sentence unsupported by the selected table cells. Annotators are restricted such that they are only able to delete phrases, transforming the original sentence: $s \rightarrow s_{\text {deletion. In Table } 1 \text {, the annotator }}$ transforms $s$ by removing several phrases such as After winning the German under-23 $100 \mathrm{~m}$ title.

On average, $\boldsymbol{s}_{\text {deletion }}$ is different from $s$ for $85.3 \%$ of examples and while $s$ has an average length of 26.6 tokens, this is reduced to 15.9 for $\boldsymbol{s}_{\text {deletion. }}$ We found that the phrases annotators often disagreed on corresponded to verbs purportedly supported by the table.

Decontextualization A given sentence $s$ may contain pronominal references or other phrases that depend on context. We thus instruct annotators to identify the main topic of the sentence; if it is a pronoun or other ambiguous phrase, we ask them to replace it with a named entity from the table or metadata. To discourage excessive modification, they are instructed to make at most one replacement. ${ }^{4}$ This transforms the sentence yet again:

\footnotetext{
${ }^{4}$ Based on manual examination of a subset of 100 examples, all of them could be decontextualized with only one replacement. Allowing annotators to make multiple replacements led to excessive clarification.
} 


\begin{tabular}{|c|c|c|c|}
\hline Original & After Deletion & After Decontextualization & Final \\
\hline $\begin{array}{l}\text { He later raced a Nissan Pulsar and } \\
\text { then a Mazda } 626 \text { in this series, with } \\
\text { a highlight of finishing runner up to } \\
\text { Phil Morriss in the } 1994 \text { Australian } \\
\text { Production Car Championship. }\end{array}$ & $\begin{array}{l}\text { He tater raced a Nissan Pulsar and } \\
\text { then a Mazda } 626 \text { in this series, with } \\
\text { a highlight of finishing runner up to } \\
\text { Phil Merriss in the } 1994 \text { Australian } \\
\text { Production Car Championship. }\end{array}$ & $\begin{array}{l}\text { Murray Carter raced a Nissan Pul- } \\
\text { sar and finished as a runner up in } \\
\text { the } 1994 \text { Australian Production Car } \\
\text { Championship. }\end{array}$ & $\begin{array}{l}\text { Murray Carter raced a Nissan Pul- } \\
\text { sar and finished as runner up in } \\
\text { the } 1994 \text { Australian Production Car } \\
\text { Championship. }\end{array}$ \\
\hline $\begin{array}{l}\text { On July } 6,2008 \text {, Webb failed to qual- } \\
\text { ify for the Beijing Olympics in the } \\
1500 \mathrm{~m} \text { after finishing } 5 \text { th in the US } \\
\text { Olympic Trials in Eugene, Oregon } \\
\text { with a time of } 3: 41.62 \text {. }\end{array}$ & $\begin{array}{l}\text { On July } 6,2008 \text {, Webb failed to } \\
\text { qualify for the Beijing Olympies in } \\
\text { the } 1500 \mathrm{~m} \text { after finishing } 5 \text { th in the } \\
\text { US Olympic Trials in Eugene, Ore- } \\
\text { gon with a time of } 3: 41.62 \text {. }\end{array}$ & $\begin{array}{l}\text { On July } 6,2008, \text { Webb finishing } \\
5 \text { th in the Olympic Trials in Eugene, } \\
\text { Oregon with a time of } 3: 41.62 \text {. }\end{array}$ & $\begin{array}{l}\text { On July 6, 2008, Webb finished 5th } \\
\text { in the Olympic Trials in Eugene, } \\
\text { Oregon, with a time of } 3: 41.62 \text {. }\end{array}$ \\
\hline $\begin{array}{l}\text { Out of the } 17,219 \text { inhabitants, } 77 \text { per- } \\
\text { cent were } 20 \text { years of age or older } \\
\text { and } 23 \text { percent were under the age of } \\
20 \text {. }\end{array}$ & $\begin{array}{l}\text { Out of the } 17,219 \text { inhabitants }, 77 \\
\text { percent were } 20 \text { years of age or older } \\
\text { and } 23 \text { pereenther the of } \\
20 \text {. }\end{array}$ & $\begin{array}{l}\text { Rawdat Al Khail had a population } \\
\text { of } 17,219 \text { inhabitants. }\end{array}$ & $\begin{array}{l}\text { Rawdat Al Khail had a population } \\
\text { of } 17,219 \text { inhabitants. }\end{array}$ \\
\hline
\end{tabular}

Table 3: Examples of annotation process. Deletions are indicated in red strikeouts, while added named entities are indicated in underlined blue. Significant grammar fixes are denoted in orange.

$\boldsymbol{s}_{\text {deletion }} \rightarrow \boldsymbol{s}_{\text {decontext }}$. In Table 1 , the annotator replaced she with Gabriele Becker.

Since the previous steps can lead to ungrammatical sentences, annotators are also instructed to fix the grammar to improve the fluency of the sentence. We find that $\boldsymbol{s}_{\text {decontext }}$ is different than $s_{\text {deletion }} 68.3 \%$ of the time, and the average sentence length increases to 17.2 tokens for $\boldsymbol{s}_{\text {decontext }}$ compared to 15.9 for $s_{\text {deletion }}$.

Secondary Annotation Task Due to the complexity of the task, $\boldsymbol{s}_{\text {decontext }}$ may still have grammatical errors, even if annotators were instructed to fix grammar. Thus, a second set of annotators were asked to further correct the sentence and were shown the table with highlighted cells as additional context. This results in the final sentence $s_{\text {final }}$. On average, annotators edited the sentence $27.0 \%$ of the time, and the sentence length slightly increased to 17.4 tokens from 17.2.

\section{Dataset Analysis}

Basic statistics of TOTTO are described in Table 5. The number of unique tables and vocabulary size attests to the open domain nature of our dataset. Furthermore, while the median table is actually quite large (87 cells), the median number of highlighted cells is significantly smaller (3). This indicates the importance of the cell highlighting feature of our dataset toward a well-defined text generation task.

\subsection{Annotator Agreement}

Table 6 shows annotator agreement over the development set for each step of the annotation process. We compute annotator agreement and Fleiss' kappa (Fleiss, 1971) for table readability and highlighted cells, and BLEU-4 score between annotated sentences in different stages.

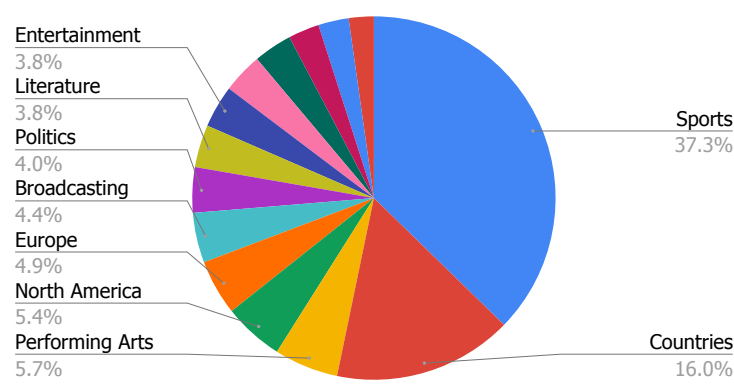

Figure 1: Topic distribution of our dataset.

As one can see, the table readability task has an agreement of $99.38 \%$. The cell highlighting task is more challenging. $73.74 \%$ of the time all three annotators completely agree on the set of cells which means that they chose the exact same set of cells. The Fleiss' kappa is 0.856 , which is regarded as "almost perfect agreement" (0.81 1.00) according to (Landis and Koch, 1977).

With respect to the sentence revision tasks, we see that the agreement slightly degrades as more steps are performed. We compute single reference BLEU among all pairs of annotators for examples in our development set (which only contains examples where both annotators chose $\boldsymbol{t}_{\text {highlight }} \neq \emptyset$ ). As the sequence of revisions are performed, the annotator agreement gradually decreases in terms of BLEU-4: $82.19 \rightarrow 72.56 \rightarrow 68.98$. This is considerably higher than the BLEU-4 between the original sentence $s$ and $s_{\text {final }}$ (43.17).

\subsection{Topics and Linguistic Phenomena}

We use the Wikimedia Foundation's topic categorization model (Asthana and Halfaker, 2018) to sort the categories of Wikipedia articles where the 
Table Title: Robert Craig (American football)

Section Title: National Football League statistics

Table Description:None

\begin{tabular}{c|c|c|c|c|c|c|c|c|c|c|c}
\hline \multicolumn{1}{c}{ RUSHING } & \multicolumn{5}{c}{ RECEIVING } \\
\hline YEAR & TEAM & ATT & YDS & AVG & LNG & TD & NO. & YDS & AVG & LNG & TD \\
\hline 1983 & SF & 176 & 725 & 4.1 & 71 & 8 & 48 & 427 & 8.9 & 23 & 4 \\
\hline 1984 & SF & 155 & 649 & 4.2 & 28 & 4 & 71 & 675 & 9.5 & 64 & 3 \\
\hline 1985 & SF & 214 & 1050 & 4.9 & 62 & 9 & 92 & 1016 & 11 & 73 & 6 \\
\hline 1986 & SF & 204 & 830 & 4.1 & 25 & 7 & 81 & 624 & 7.7 & 48 & 0 \\
\hline 1987 & SF & 215 & 815 & 3.8 & 25 & 3 & 66 & 492 & 7.5 & 35 & 1 \\
\hline 1988 & SF & 310 & 1502 & 4.8 & 46 & 9 & 76 & 534 & 7.0 & 22 & 1 \\
\hline 1989 & SF & 271 & 1054 & 3.9 & 27 & 6 & 49 & 473 & 9.7 & 44 & 1 \\
\hline 1990 & SF & 141 & 439 & 3.1 & 26 & 1 & 25 & 201 & 8.0 & 31 & 0 \\
\hline 1991 & RAI & 162 & 590 & 3.6 & 15 & 1 & 17 & 136 & 8.0 & 20 & 0 \\
\hline 1992 & MIN & 105 & 416 & 4.0 & 21 & 4 & 22 & 164 & 7.5 & 22 & 0 \\
\hline 1993 & MIN & 38 & 119 & 3.1 & 11 & 1 & 19 & 169 & 8.9 & 31 & 1 \\
\hline Totals & - & $\mathbf{1 9 9 1}$ & $\mathbf{8 1 8 9}$ & $\mathbf{4 . 1}$ & $\mathbf{7 1}$ & $\mathbf{5 6}$ & $\mathbf{5 6 6}$ & $\mathbf{4 9 1 1}$ & $\mathbf{8 . 7}$ & $\mathbf{7 3}$ & $\mathbf{1 7}$ \\
\hline Target Text: Craig finished his eleven NFL seasons with 8,189 rushing yards and 566 receptions for 4,911 receiving yards.
\end{tabular}

Table 4: An example in the TOTTO dataset that involves numerical reasoning over the table structure.

\begin{tabular}{lr}
\hline Property & Value \\
\hline Training set size & 120,761 \\
Number of target tokens & $1,268,268$ \\
Avg Target Length (tokens) & 17.4 \\
Target vocabulary size & 136,777 \\
Unique Tables & 83,141 \\
Rows per table (Median/Avg) & $16 / 32.7$ \\
Cells per table (Median/Avg) & $87 / 206.6$ \\
No. of Highlighted Cell (Median/Avg) & $3 / 3.55$ \\
\hline Development set size & 7,700 \\
Test set size & 7,700 \\
\hline
\end{tabular}

Table 5: ToTTo dataset statistics.

\begin{tabular}{llr}
\hline Annotation Stage & Measure & Result \\
\hline Table Readability & Agreement / $\kappa$ & 99.38 / 0.646 \\
Cell Highlighting & Agreement / $\kappa$ & 73.74 / 0.856 \\
After Deletion & BLEU-4 & 82.19 \\
After Decontextualization & BLEU-4 & 72.56 \\
Final & BLEU-4 & 68.98 \\
\hline
\end{tabular}

Table 6: Annotator agreement over the development set. If possible, we measure the total agreement (in \%) and the Fleiss' Kappa $(\kappa)$. Otherwise, we report the BLEU4 between annotators.

tables come from into a 44-category ontology. ${ }^{5}$ Figure 1 presents an aggregated topic analysis of our dataset. We found that the Sports and Countries topics together comprise $53.4 \%$ of our dataset, but the other $46.6 \%$ is composed of broader topics such as Performing Arts, Politics, and North America. Our dataset is limited to topics that are present in Wikipedia.

Table 7 summarizes the fraction of examples that require reference to the metadata, as well as some of the challenging linguistic phenomena in the dataset that potentially pose new challenges to

\footnotetext{
${ }^{5}$ https://en.wikipedia.org/wiki/ Wikipedia:WikiProject_Council/Directory
}

\begin{tabular}{lr}
\hline Types & Percentage \\
\hline Require reference to page title & $82 \%$ \\
Require reference to section title & $19 \%$ \\
Require reference to table description & $3 \%$ \\
Reasoning (logical, numerical, temporal etc.) & $21 \%$ \\
Comparison across rows / columns / cells & $13 \%$ \\
Require background information & $12 \%$ \\
\hline
\end{tabular}

Table 7: Distribution of different linguistic phenomena among 100 randomly chosen sentences.

current systems. Table 4 gives one example that requires reasoning (refer to the Appendix for more examples).

\subsection{Training, Development, and Test Splits}

After the annotation process, we only consider examples where the sentence is related to the table, i.e., $\boldsymbol{t}_{\text {highlight }} \neq \emptyset$. This initially results in a training set $\boldsymbol{D}_{\text {orig-train }}$ of size 131,849 that we further filter as described below. Each example in the development and test sets was annotated by three annotators. Since the machine learning task uses $\boldsymbol{t}_{\text {highlight }}$ as an input, it is challenging to use three different sets of highlighted cells in evaluation. Thus, we only use a single randomly chosen $\boldsymbol{t}_{\text {highlight }}$ while using the three $s_{\text {final }}$ as references for evaluation. We only use examples where at least 2 of the 3 annotators chose $\boldsymbol{t}_{\text {highlight }} \neq \emptyset$, resulting in development and test sets of size 7,700 each.

Overlap and Non-Overlap Sets Without any modification $\boldsymbol{D}_{\text {orig-train }}, \boldsymbol{D}_{\text {dev }}$, and $\boldsymbol{D}_{\text {test }}$ may contain many similar tables. Thus, to increase the generalization challenge, we filter $\boldsymbol{D}_{\text {orig-train }}$ to remove some examples based on overlap with $\boldsymbol{D}_{\text {dev }}, \boldsymbol{D}_{\text {test }}$.

For a given example $\boldsymbol{d}$, let $\boldsymbol{h}(\boldsymbol{d})$ denote its set of header values and similarly let $\boldsymbol{h}(\boldsymbol{D})$ be the set of 
header values for a given dataset $\boldsymbol{D}$. We remove examples $\boldsymbol{d}$ from the training set where $\boldsymbol{h}(\boldsymbol{d})$ is both rare in the data as well as occurs in either the development or test sets. Specifically, $\boldsymbol{D}_{\text {train }}$ is defined as:

$$
\begin{array}{r}
\boldsymbol{D}_{\text {train }}:=\left\{\boldsymbol{d}: \boldsymbol{h}(\boldsymbol{d}) \notin\left(\boldsymbol{h}\left(\boldsymbol{D}_{\text {dev }}\right) \cup \boldsymbol{h}\left(\boldsymbol{D}_{\text {test }}\right)\right)\right. \text { or } \\
\text { count } \left.\left(\boldsymbol{h}(\boldsymbol{d}), \boldsymbol{D}_{\text {orig-train }}\right)>\alpha\right\} .
\end{array}
$$

The count $\left(\boldsymbol{h}(\boldsymbol{d}), \boldsymbol{D}_{\text {orig-train }}\right)$ function returns the number of examples in $\boldsymbol{D}_{\text {orig-train }}$ with header $\boldsymbol{h}(\boldsymbol{d})$. To choose the hyperparameter $\alpha$ we first split the test set as follows:

$$
\begin{aligned}
\boldsymbol{D}_{\text {test-overlap }}:=\left\{\boldsymbol{d}: \boldsymbol{h}(\boldsymbol{d}) \in \boldsymbol{h}\left(\boldsymbol{D}_{\text {train }}\right)\right\} \\
\boldsymbol{D}_{\text {test-nonoverlap }}:=\left\{\boldsymbol{d}: \boldsymbol{h}(\boldsymbol{d}) \notin \boldsymbol{h}\left(\boldsymbol{D}_{\text {train }}\right)\right\}
\end{aligned}
$$

The development set is analogously divided into $\boldsymbol{D}_{\text {dev-overlap }}$ and $\boldsymbol{D}_{\text {dev-nonoverlap. We then choose }}$ $\alpha=5$ so that $\boldsymbol{D}_{\text {test-overlap }}$ and $\boldsymbol{D}_{\text {test-nonoverlap }}$ have similar size. After filtering, the size of $\boldsymbol{D}_{\text {train }}$ is 120,761 , and $\boldsymbol{D}_{\text {dev-overlap }}, \boldsymbol{D}_{\text {dev-nonoverlap }}$, $\boldsymbol{D}_{\text {test-overlap }}$, and $\boldsymbol{D}_{\text {test-nonoverlap }}$ have sizes 3784 , 3916,3853 , and 3847 respectively.

\section{Machine Learning Task Construction}

In this work, we focus on the following task: Given a table $\boldsymbol{t}$, related metadata $\boldsymbol{m}$ (page title, section title, table section text) and a set of highlighted cells $t_{\text {highlight }}$, produce the final sentence $s_{\text {final }}$. Mathematically this can be described as learning a function $\boldsymbol{f}: \boldsymbol{x} \rightarrow \boldsymbol{y}$ where $\boldsymbol{x}=\left(\boldsymbol{t}, \boldsymbol{m}, \boldsymbol{t}_{\text {highlight }}\right)$ and $\boldsymbol{y}=\boldsymbol{s}_{\text {final }}$. This task is different from what the annotators perform, since they are provided a starting sentence requiring revision. Therefore, the task is more challenging, as the model must generate a new sentence instead of revising an existing one.

\section{Experiments}

We present baseline results on TOTTO by examining three existing state-of-the-art approaches (Note that since our tables do not have a fixed schema it is difficult to design a template baseline).

- BERT-to-BERT (Rothe et al., 2020): A Transformer encoder-decoder model (Vaswani et al., 2017) where the encoder and decoder are both initialized with BERT (Devlin et al., 2018). The original BERT model is pre-trained with both Wikipedia and the Books corpus (Zhu et al., 2015), the former of which contains our (unrevised) test targets. Thus, we also pre-train a version of BERT on the Books corpus only, which we consider a more correct baseline. However, empirically we find that both models perform similarly in practice (Table 8).

- Pointer-Generator (See et al., 2017): A Seq2Seq model with attention and copy mechanism. While originally designed for summarization it is commonly used in data-to-text as well (Gehrmann et al., 2018).

- Puduppully et al. (2019): A Seq2Seq model with an explicit content selection and planning mechanism designed for data-to-text.

Details about hyperparameter settings are provided in the Appendix. Moreover, we explore different strategies of representing the source content that resemble standard linearization approaches in the literature (Lebret et al., 2016; Wiseman et al., 2017)

- Full Table The simplest approach is simply to use the entire table as the source, adding special tokens to mark which cells have been highlighted. However, many tables can be very large and this strategy performs poorly.

- Subtable Another option is to only use the highlighted cells $\boldsymbol{t}_{\text {highlight }} \in \boldsymbol{t}$ with the heuristically extracted row and column header for each highlighted cell. This makes it easier for the model to only focus on relevant content but limits the ability to perform reasoning in the context of the table structure (see Table 11). Overall though, we find this representation leads to higher performance.

In all cases, the cells are linearized with row and column separator tokens. We also experiment with prepending the table metadata to the source table. ${ }^{6}$

Evaluation metrics The model output is evaluated using two automatic metrics: BLEU (Papineni et al., 2002) and PARENT (Dhingra et al., 2019). PARENT is a metric recently proposed specifically for data-to-text evaluation that takes the table into account. We modify it to make it suitable for our dataset, described in the Appendix. Human evaluation is described in $\S 8.2$.

\subsection{Results}

Table 8 shows our results against multiple references with the subtable input format. Both the

\footnotetext{
${ }^{6}$ The table section text is ignored, since it is usually missing or irrelevant.
} 


\begin{tabular}{lrrrrrr}
\hline \multirow{2}{*}{ Model } & \multicolumn{2}{c}{ Overall } & \multicolumn{2}{c}{ Overlap Subset } & \multicolumn{2}{c}{ Nonoverlap Subset } \\
\cline { 2 - 6 } & BLEU & PARENT & BLEU & PARENT & BLEU & PARENT \\
\hline BERT-to-BERT (Books+Wiki) & $\mathbf{4 4 . 0}$ & $\mathbf{5 2 . 6}$ & $\mathbf{5 2 . 7}$ & $\mathbf{5 8 . 4}$ & $\mathbf{3 5 . 1}$ & $\mathbf{4 6 . 8}$ \\
BERT-to-BERT (Books) & 43.9 & $\mathbf{5 2 . 6}$ & $\mathbf{5 2 . 7}$ & $\mathbf{5 8 . 4}$ & 34.8 & 46.7 \\
Pointer-Generator & 41.6 & 51.6 & 50.6 & 58.0 & 32.2 & 45.2 \\
Puduppully et al. (2019) & 19.2 & 29.2 & 24.5 & 32.5 & 13.9 & 25.8 \\
\hline
\end{tabular}

Table 8: Performance compared to multiple references on the test set for the subtable input format with metadata.

\begin{tabular}{|c|c|c|c|c|c|}
\hline & Model & Fluency $(\%)$ & Faithfulness (\%) & Covered Cells $(\%)$ & Less/Neutral/More Coverage w.r.t. Ref \\
\hline \multirow{3}{*}{ Overall } & Oracle & 99.3 & 93.6 & 94.8 & $18.3 / 61.7 / 20.0$ \\
\hline & BERT-to-BERT (Books) & 88.1 & 76.2 & 89.0 & $49.2 / 36.2 / 14.5$ \\
\hline & BERT-to-BERT (Books+Wiki) & 87.3 & 73.6 & 87.3 & $53.9 / 32.9 / 13.2$ \\
\hline \multirow{3}{*}{ Overlap } & Oracle & 99.6 & 96.5 & 95.5 & $19.8 / 62.8 / 17.4$ \\
\hline & BERT-to-BERT (Books) & 89.6 & 78.7 & 92.1 & $42.0 / 43.7 / 14.3$ \\
\hline & BERT-to-BERT (Books+Wiki) & 89.8 & 81.1 & 91.0 & $47.8 / 39.2 / 13.1$ \\
\hline \multirow{3}{*}{ Non-overlap } & Oracle & 99.1 & 91.4 & 94.3 & $17.0 / 60.9 / 22.1$ \\
\hline & BERT-to-BERT (Books) & 86.9 & 74.2 & 86.4 & $55.5 / 29.8 / 14.7$ \\
\hline & BERT-to-BERT (Books+Wiki) & 84.8 & 66.6 & 83.8 & $60.1 / 26.6 / 13.3$ \\
\hline
\end{tabular}

Table 9: Human evaluation over references (to compute Oracle) and model outputs. For Fluency, we report the percentage of outputs that were completely fluent. In the last column $X / Y / Z$ means $\mathrm{X} \%$ and $\mathrm{Z} \%$ of the candidates were deemed to be less and more informative than the reference respectively and Y\% were neutral.

\begin{tabular}{lrr}
\hline Data Format & BLEU & PARENT \\
\hline subtable w/ metadata & 43.9 & 52.6 \\
subtable w/o metadata & 36.9 & 42.6 \\
full table w/ metadata & 26.8 & 30.7 \\
full table w/o metadata & 20.9 & 22.2 \\
\hline
\end{tabular}

Table 10: Multi-reference performance of different input representations for BERT-to-BERT Books model.

BERT-to-BERT models perform the best, followed by the pointer generator model. ${ }^{7}$ We see that for all models the performance on the non-overlap set is significantly lower than that of the overlap set, indicating that slice of our data poses significant challenges for machine learning models. We also observe that the baseline that separates content selection and planning performs quite poorly. We attest this to the fact that it is engineered to the Rotowire data format and schema.

Table 10 explores the effects of the various input representations (subtable vs. full table) on the BERT-to-BERT model. We see that the full table format performs poorly even if it is the most knowledge-preserving representation.

\subsection{Human evaluation}

For each of the 2 top performing models in Table 8, we take 500 random outputs and perform human evaluation using the following axes:

- Fluency - A candidate sentence is fluent if it is grammatical and natural. The three choices are Fluent, Mostly Fluent, Not Fluent.

\footnotetext{
${ }^{7}$ Note the BLEU scores are relatively high due to the fact that our task is more controlled than other text generation tasks and that we have multiple references.
}

- Faithfulness (Precision) - A candidate sentence is considered faithful if all pieces of information are supported by either the table or one of the references. Any piece of unsupported information makes the candidate unfaithful.

- Covered Cells (Recall) - Percentage of highlighted cells the candidate sentence covers.

- Coverage with Respect to Reference (Recall) - We ask whether the candidate is strictly more or less informative than each reference (or neither, which is referred to as neutral).

We further compute an oracle upper-bound by treating one of the references as a candidate and evaluating it compared to the table and other references. The results, shown in Table 9, attest to the high quality of our human annotations since the oracle consistently achieves high performance. All the axes demonstrate that there is a considerable gap between the model and oracle performance.

This difference is most easily revealed in the last column when annotators are asked to directly compare the candidate and reference. As expected, the oracle has similar coverage to the reference $(61.7 \%$ neutral) but both baselines demonstrate considerably less coverage. According to an independentsample t-test, this difference is significant at a $p<0.001$ level for both baselines. Furthermore, the baselines are considerably less faithful than the reference. The faithfulness of both models is significantly lower than the reference ( $\chi^{2}$ test with $p<0.001)$. The models do not differ significantly from each other, except for faithfulness 


\begin{tabular}{|c|c|c|c|c|}
\hline ID & Reference & \multicolumn{2}{|c|}{ Decoder output (w/ metadata) } & w/o metadata \\
\hline & & Full table & Subtable & Subtable \\
\hline 1 & $\begin{array}{l}\text { in the } 1939 \text { currie cup, western } \\
\text { province lost to transvaal by } 176 \text { in } \\
\text { cape town. }\end{array}$ & $\begin{array}{l}\text { northern transvaal and western } \\
\text { province were drawn by } 16 \\
\text { in } 1989 \text { and in } 1995 \text {, western } \\
\text { province were defeated by the } \\
\text { sharks in durban. }\end{array}$ & $\begin{array}{l}\text { the first currie cup was played } \\
\text { in } 1939 \text { in transvaal } 1 \text { at newlands, } \\
\text { with western province winning } 176 .\end{array}$ & $\begin{array}{l}\text { the first scottish cup was played } \\
\text { in } 1939 \text { in transvaal1, where } \\
\text { transvaal1 defeated western } \\
\text { province } 176 \text { in the final at new- } \\
\text { lands in cape town. }\end{array}$ \\
\hline 2 & $\begin{array}{l}\text { a second generation of microdrive } \\
\text { was announced by ibm in } 2000 \text { with } \\
\text { increased capacities at } 512 \mathrm{mb} \text { and } 1 \\
\text { gb. }\end{array}$ & $\begin{array}{l}\text { the microdrive models formed } 512 \\
\text { megabyte and } 1 \text { gigabyte in } 2000 .\end{array}$ & $\begin{array}{l}\text { there were } 512 \text { microdrive models } \\
\text { in 2000: } 1 \text { gigabyte. }\end{array}$ & $\begin{array}{l}\begin{array}{l}\text { cortete's production } \\
\text { megabyte. }\end{array}\end{array}$ \\
\hline 3 & $\begin{array}{l}\text { the } 1956 \text { grand prix motorcycle rac- } \\
\text { ing season consisted of six grand } \\
\text { prix races in five classes: } 500 \mathrm{cc} \text {, } \\
350 \mathrm{cc}, 250 \mathrm{cc}, 125 \mathrm{cc} \text { and sidecars } \\
500 \mathrm{cc} \text {. }\end{array}$ & $\begin{array}{l}\text { the } 1966 \text { grand prix motorcycle } \\
\text { racing season consisted of seven } \\
\text { grand prix races in five classes: } \\
500 \mathrm{cc}, 350 \mathrm{cc}, 250 \mathrm{cc}, 125 \mathrm{cc} \text { and } \\
\text { sidecars } 500 \mathrm{cc} \text {. }\end{array}$ & $\begin{array}{l}\text { the } 1956 \text { grand prix motorcycle rac- } \\
\text { ing season consisted of eight grand } \\
\text { prix races in five classes: } 500 \mathrm{cc} \text {, } \\
350 \mathrm{cc}, 250 \mathrm{cc}, 125 \mathrm{cc} \text { and sidecars } \\
500 \mathrm{cc} \text {. }\end{array}$ & $\begin{array}{l}\text { the } 1955 \text { grand prix motorcycle rac- } \\
\text { ing season consisted of eight grand } \\
\text { prix races in five classes: 500cc, } \\
350 \mathrm{cc}, 250 \mathrm{cc}, 125 \mathrm{cc} \text { and sidecars } \\
500 \mathrm{cc} \text {. }\end{array}$ \\
\hline 4 & $\begin{array}{l}\text { in travis kelce's last collegiate sea- } \\
\text { son, he set personal career highs } \\
\text { in receptions ( } 45 \text { ), receiving yards } \\
\text { ( } 722) \text {, yards per receptions (16.0) } \\
\text { and receiving touchdowns (8). }\end{array}$ & $\begin{array}{l}\text { during the } 2011 \text { season, travis kel- } \\
\text { ceum caught } 76 \text { receptions for } \\
1,612 \text { yards and } 14 \text { touchdowns. }\end{array}$ & $\begin{array}{l}\text { travis kelce finished the } 2012 \text { sea- } \\
\text { son with } 45 \text { receptions for } 722 \\
\text { yards }(16.0 \text { avg. }) \text { and eight touch- } \\
\text { downs. }\end{array}$ & $\begin{array}{l}\text { kelce finished the } 2012 \text { season with } \\
45 \text { catches for } 722 \text { yards ( } 16.0 \text { avg.) } \\
\text { and eight touchdowns. }\end{array}$ \\
\hline 5 & $\begin{array}{l}\text { in the } 2012 \text { film pizza bagel, michael } \\
\text { pillarella portrays tommy. }\end{array}$ & $\begin{array}{l}\text { in } 2012 \text {, groff played the role of } \\
\text { tommy in the film pizza bagel. }\end{array}$ & $\begin{array}{l}\text { in } 2012 \text {, pillarella appeared as } \\
\text { tommy in the film pizza bagel. }\end{array}$ & $\begin{array}{l}\text { harris played the role of tommy in } \\
\text { the } 2012 \text { film pizza bagel. }\end{array}$ \\
\hline 6 & $\begin{array}{l}\text { the album shari addison placed at no. } \\
176 \text { on the billboard } 200 \text { along with } \\
\text { no. } 5 \text { on the gospel albums. }\end{array}$ & $\begin{array}{l}\text { shari addison's " 5", reached num- } \\
\text { ber } 176 \text { on the billboard } 200 .\end{array}$ & $\begin{array}{l}\text { shari addison charted at number } 176 \\
\text { on the us chart and at number } 5 \text { on } \\
\text { the us billboard } 200 \text {. }\end{array}$ & $\begin{array}{l}\text { the shari addison peaked at number } \\
176 \text { on the billboard } 200 \text { chart. }\end{array}$ \\
\hline
\end{tabular}

Table 11: Decoder output examples from BERT-to-BERT Books models on the development set. The "subtable with metadata" model achieves the highest BLEU. Red indicates model errors and blue denotes interesting reference language not in the model output.

in the non-overlap case, where we see a moderate effect favoring the book model.

\section{Model Errors and Challenges}

Table 11 shows predictions from the BERT-toBERT Books model to illustrate challenges existing models face.

Hallucination The model sometimes outputs phrases such as first, winning that seem reasonable but are not faithful to the table. This hallucination phenomenon has been widely observed in other existing data-to-text datasets (Lebret et al., 2016; Wiseman et al., 2017). However, the noisy references in these datasets make it difficult to disentangle model incapability from data noise. Our dataset serves as strong evidence that even when the reference targets are faithful to the source, neural models still struggle with faithfulness.

Rare topics Another challenge revealed by the open domain nature of our task is rare or complex topics at the tail of the topic distribution (Figure 1). For instance, example 2 of Table 11 concerns microdrive capacities which is challenging.

Diverse table structure and numerical reasoning In example 3, inferring six and five correctly requires counting table rows and columns. Similarly, in example 4, the phrases last and career highs can be deduced from the table structure and with comparisons over the columns. However, the model is unable to make these inferences from the simplistic source representation that we used.

Evaluation metrics Many of the above issues are difficult to capture with metrics like BLEU since the reference and prediction may only differ by a word but largely differ in terms of semantic meaning. This urges for better metrics possibly built on learned models (Wiseman et al., 2017; Ma et al., 2019; Sellam et al., 2020). Thus, while we have a task leaderboard, it should not be interpreted as the definitive measure of model performance.

\section{Conclusion}

We presented ToTTO, a table-to-text dataset that presents a controlled generation task and a data annotation process based on iterative sentence revision. We also provided several state-of-the-art baselines, and demonstrated TOTTO could serve as a useful research benchmark for model and metric development. TOTTO is available at https://github.com/ google-research-datasets/totto.

\section{Acknowledgements}

The authors wish to thank Ming-Wei Chang, Jonathan H. Clark, Kenton Lee, and Jennimaria Palomaki for their insightful discussions and support. Many thanks also to Ashwin Kakarla and his team for help with the annotations. 


\section{References}

Sumit Asthana and Aaron Halfaker. 2018. With few eyes, all hoaxes are deep. Proceedings of the ACM on Human-Computer Interaction, 2(CSCW):21.

Dzmitry Bahdanau, Kyunghyun Cho, and Yoshua Bengio. 2014. Neural machine translation by jointly learning to align and translate. In Proc. of EMNLP.

Eva Banik, Claire Gardent, and Eric Kow. 2013. The kbgen challenge. In Proc. of European Workshop on $N L G$.

David L Chen and Raymond J Mooney. 2008. Learning to sportscast: a test of grounded language acquisition. In Proc. of ICML.

Wenhu Chen, Jianshu Chen, Yu Su, Zhiyu Chen, and William Yang Wang. 2020. Logical natural language generation from open-domain tables. In Proc. of $A C L$.

Wenhu Chen, Hongmin Wang, Jianshu Chen, Yunkai Zhang, Hong Wang, Shiyang Li, Xiyou Zhou, and William Yang Wang. 2019. TabFact: A large-scale dataset for table-based fact verification. In Proc. of $I C L R$.

Jacob Devlin, Ming-Wei Chang, Kenton Lee, and Kristina Toutanova. 2018. BERT: Pre-training of deep bidirectional transformers for language understanding. In Proc. of NAACL.

Bhuwan Dhingra, Manaal Faruqui, Ankur Parikh, Ming-Wei Chang, Dipanjan Das, and William W Cohen. 2019. Handling divergent reference texts when evaluating table-to-text generation. In Proc. of $A C L$.

Dheeru Dua, Yizhong Wang, Pradeep Dasigi, Gabriel Stanovsky, Sameer Singh, and Matt Gardner. 2019. DROP: A reading comprehension benchmark requiring discrete reasoning over paragraphs. In Proc. of $N A A C L$.

John Duchi, Elad Hazan, and Yoram Singer. 2011. Adaptive subgradient methods for online learning and stochastic optimization.

Joseph L. Fleiss. 1971. Measuring nominal scale agreement among many raters. Psychological Bulletin, 76(5):378.

Claire Gardent, Anastasia Shimorina, Shashi Narayan, and Laura Perez-Beltrachini. 2017a. Creating training corpora for NLG micro-planning. In Proc. of $A C L$.

Claire Gardent, Anastasia Shimorina, Shashi Narayan, and Laura Perez-Beltrachini. 2017b. The WebNLG challenge: Generating text from RDF data. In Proc. of INLG.

Sebastian Gehrmann, Falcon Z Dai, Henry Elder, and Alexander M Rush. 2018. End-to-end content and plan selection for data-to-text generation. In INLG.
Suchin Gururangan, Swabha Swayamdipta, Omer Levy, Roy Schwartz, Samuel R Bowman, and Noah A Smith. 2018. Annotation artifacts in natural language inference data. In Proc. of NAACL.

Kelvin Guu, Tatsunori B Hashimoto, Yonatan Oren, and Percy Liang. 2018. Generating sentences by editing prototypes. TACL, 6:437-450.

Diederik P Kingma and Jimmy Ba. 2015. Adam: A method for stochastic optimization. In Proc. of ICLR.

Philipp Koehn and Rebecca Knowles. 2017. Six challenges for neural machine translation. In Proc. of WMT.

Flip Korn, Xuezhi Wang, You Wu, and Cong Yu. 2019. Automatically generating interesting facts from wikipedia tables. In Proceedings of the 2019 International Conference on Management of Data, SIGMOD 19, page 349361, New York, NY, USA. Association for Computing Machinery.

Wojciech Kryściński, Nitish Shirish Keskar, Bryan McCann, Caiming Xiong, and Richard Socher. 2019. Neural text summarization: A critical evaluation. In Proc. of EMNLP.

Karen Kukich. 1983. Design of a knowledge-based report generator. In Proc. of ACL.

J. Richard Landis and Gary G. Koch. 1977. The measurement of observer agreement for categorical data. Biometrics, 33(1):159-174.

Rémi Lebret, David Grangier, and Michael Auli. 2016. Neural text generation from structured data with application to the biography domain. In Proc. of EMNLP.

Katherine Lee, Orhan Firat, Ashish Agarwal, Clara Fannjiang, and David Sussillo. 2018. Hallucinations in neural machine translation. In Open Review.

Percy Liang, Michael I Jordan, and Dan Klein. 2009. Learning semantic correspondences with less supervision. In Proc. of ACL.

Greg Little, Lydia B Chilton, Max Goldman, and Robert C Miller. 2010. Turkit: human computation algorithms on mechanical turk. In Proceedings of the 23nd annual ACM symposium on User interface software and technology, pages 57-66.

Tianyu Liu, Kexiang Wang, Lei Sha, Baobao Chang, and Zhifang Sui. 2018. Table-to-text generation by structure-aware seq2seq learning. In Proc. of AAAI.

Qingsong Ma, Johnny Wei, Ondřej Bojar, and Yvette Graham. 2019. Results of the WMT19 metrics shared task: Segment-level and strong mt systems pose big challenges. In Proceedings of the Fourth Conference on Machine Translation (Volume 2: Shared Task Papers, Day 1), pages 62-90. 
Inderjeet Mani. 1999. Advances in automatic text summarization. MIT press.

Kathleen McKeown. 1992. Text generation. Cambridge University Press.

Jekaterina Novikova, Ondřej Dušek, and Verena Rieser. 2017. The E2E dataset: New challenges for end-toend generation. In Proc. of SIGDIAL.

Jekaterina Novikova, Oliver Lemon, and Verena Rieser. 2016. Crowd-sourcing nlg data: Pictures elicit better data. In Proc. of INLG.

Gaurav Pandey, Danish Contractor, Vineet Kumar, and Sachindra Joshi. 2018. Exemplar encoder-decoder for neural conversation generation. In Proc. of $A C L$.

Kishore Papineni, Salim Roukos, Todd Ward, and WeiJing Zhu. 2002. BLEU: a method for automatic evaluation of machine translation. In Proc. of ACL.

Panupong Pasupat and Percy Liang. 2015. Compositional semantic parsing on semi-structured tables. In Proc. of ACL.

Hao Peng, Ankur P Parikh, Manaal Faruqui, Bhuwan Dhingra, and Dipanjan Das. 2019. Text generation with exemplar-based adaptive decoding. In Proc. of $N A A C L$.

Laura Perez-Beltrachini and Mirella Lapata. 2018. Bootstrapping generators from noisy data. In Proc. of NAACL.

Adam Poliak, Jason Naradowsky, Aparajita Haldar, Rachel Rudinger, and Benjamin Van Durme. 2018. Hypothesis only baselines in natural language inference. In*SEM@NAACL-HLT.

Ratish Puduppully, Li Dong, and Mirella Lapata. 2019. Data-to-text generation with content selection and planning. In Proc. of AAAI.

Ehud Reiter and Robert Dale. 1997. Building applied natural language generation systems. Natural Language Engineering, 3(1):57-87.

Sascha Rothe, Shashi Narayan, and Aliaksei Severyn. 2020. Leveraging pre-trained checkpoints for sequence generation tasks. In Proc. of TACL.

Abigail See, Peter J. Liu, and Christopher D. Manning. 2017. Get to the point: Summarization with pointergenerator networks. In Proc. of $A C L$.

Thibault Sellam, Dipanjan Das, and Ankur P Parikh. 2020. BLEURT: Learning robust metrics for text generation. In Proc. of ACL.

Ilya Sutskever, Oriol Vinyals, and Quoc V Le. 2014. Sequence to sequence learning with neural networks. In Proc. of NIPS.

Ran Tian, Shashi Narayan, Thibault Sellam, and Ankur P Parikh. 2019. Sticking to the facts: Confident decoding for faithful data-to-text generation. arXiv preprint arXiv:1910.08684.
Ashish Vaswani, Noam Shazeer, Niki Parmar, Jakob Uszkoreit, Llion Jones, Aidan N Gomez, Łukasz Kaiser, and Illia Polosukhin. 2017. Attention is all you need. In Proc. of NIPS.

Oriol Vinyals and Quoc Le. 2015. A neural conversational model. In Proc. of ICML Deep Learning Workshop.

Tsung-Hsien Wen, Milica Gasic, Nikola Mrksic, PeiHao Su, David Vandyke, and Steve Young. 2015. Semantically conditioned LSTM-based natural language generation for spoken dialogue systems. In Proc. of EMNLP.

Sam Wiseman, Stuart M Shieber, and Alexander M Rush. 2017. Challenges in data-to-document generation. In Proc. of EMNLP.

Yukun Zhu, Ryan Kiros, Rich Zemel, Ruslan Salakhutdinov, Raquel Urtasun, Antonio Torralba, and Sanja Fidler. 2015. Aligning books and movies: Towards story-like visual explanations by watching movies and reading books. In Proc. of ICCV.

\section{A Appendix}

The Appendix contains the following contents:

- Information about the variant of the PARENT metric (Dhingra et al., 2019) used for evaluation.

- More details about the baselines.

- Examples of more complex tables in our dataset (Figure 2-Figure 5).

\section{A.1 PARENT metric}

PARENT (Dhingra et al., 2019) is a metric recently proposed specifically for data-to-text evaluation that takes the table into account. We modify it to make it suitable for our dataset. Let $\left(\boldsymbol{x}_{n}, \boldsymbol{y}_{n}, \hat{\boldsymbol{y}}_{n}\right)$ denote one example that consists of a (source, target, prediction) tuple. PARENT is defined at an instance level as:

$$
\begin{aligned}
& P A R E N T\left(\boldsymbol{x}_{n}, \boldsymbol{y}_{n}, \hat{\boldsymbol{y}}_{n}\right)= \\
& \frac{2 \times E_{p}\left(\boldsymbol{x}_{n}, \boldsymbol{y}_{n}, \hat{\boldsymbol{y}}_{n}\right) \times E_{r}\left(\boldsymbol{x}_{n}, \boldsymbol{y}_{n}, \hat{\boldsymbol{y}}_{n}\right)}{E_{p}\left(\boldsymbol{x}_{n}, \boldsymbol{y}_{n}, \hat{\boldsymbol{y}}_{n}\right)+E_{r}\left(\boldsymbol{x}_{n}, \boldsymbol{y}_{n}, \hat{\boldsymbol{y}}_{n}\right)}
\end{aligned}
$$

$E_{p}\left(\boldsymbol{x}_{n}, \boldsymbol{y}_{n}, \hat{\boldsymbol{y}}_{n}\right)$ is the PARENT precision computed using the prediction, reference, and table (the last of which is not used in BLEU). $E_{r}\left(\boldsymbol{x}_{n}, \boldsymbol{y}_{n}, \hat{\boldsymbol{y}}_{n}\right)$ is the PARENT recall and is computed as:

$$
E_{r}\left(\boldsymbol{x}_{n}, \boldsymbol{y}_{n}, \hat{\boldsymbol{y}}_{n}\right)=R\left(\boldsymbol{x}_{n}, \boldsymbol{y}_{n}, \hat{\boldsymbol{y}}_{n}\right)^{(1-\lambda)} R\left(\boldsymbol{x}_{n}, \hat{\boldsymbol{y}}_{n}\right)^{\lambda}
$$

where $R\left(\boldsymbol{x}_{n}, \boldsymbol{y}_{n}, \hat{\boldsymbol{y}}_{n}\right)$ is a recall term that compares the prediction with both the reference and table. 
$R\left(\boldsymbol{x}_{n}, \hat{\boldsymbol{y}}_{n}\right)$ is an extra recall term that gives an additional reward if the prediction $\hat{\boldsymbol{y}}_{n}$ contains phrases in the table $\boldsymbol{x}_{n}$ that are not necessarily in the reference ( $\lambda$ is a hyperparameter).

In the original PARENT work, the same table $t$ is used for computing the precision and both recall terms. While this makes sense for most existing datasets, it does not take into account the highlighted cells $\boldsymbol{t}_{\text {highlight }}$ in our task. To incorporate $\boldsymbol{t}_{\text {highlight }}$, we modify the PARENT metric so that the additional recall term $R\left(\boldsymbol{x}_{n}, \hat{\boldsymbol{y}}_{n}\right)$ uses $\boldsymbol{t}_{\text {highlight }}$ instead of $\boldsymbol{t}$ to only give an additional reward for relevant table information. The other recall and the precision term still use $\boldsymbol{t}$.

\section{A.2 Baseline details}

- BERT-to-BERT (Rothe et al., 2020) - Uncased model coupling both encoder and decoder as in original paper, with Adam optimizer (Kingma and $\mathrm{Ba}, 2015)$. learning rate $=$ 0.05 , hidden size $=1024$, dropout $=0.1$, beam size $=4$.

- Pointer Generator (See et al., 2017) - LSTM with hidden size 300 , beam size $=8$, learning rate $=0.0003$, dropout $=0.2$, length penalty $=$ 0.0, Adam optimizer (Kingma and Ba, 2015).

- Content planner (Puduppully et al., 2019) - All of the original hyperparameters: content planner: LSTM with hidden size $1 \times 600$, realizer LSTM with $2 \times 600$, embedding size 600 for both, dropout $=0.3$, Adagrad optimizer (Duchi et al., 2011), beam size $=5$. 
Table Title: Ken Fujita

Section Title: Club statistics

Table Description: None

\begin{tabular}{|c|c|c|c|c|c|c|c|c|c|c|}
\hline \multicolumn{3}{|c|}{ Club performance } & \multicolumn{2}{|c|}{ League } & \multicolumn{2}{|c|}{ Cup } & \multicolumn{2}{|c|}{ League Cup } & \multicolumn{2}{|c|}{ Total } \\
\hline Season & Club & League & Apps & Goals & Apps & Goals & Apps & Goals & Apps & Goals \\
\hline \multicolumn{3}{|c|}{ Japan } & \multicolumn{2}{|c|}{ League } & \multicolumn{2}{|c|}{ Emperor's Cup } & \multicolumn{2}{|c|}{ J.League Cup } & \multicolumn{2}{|c|}{ Total } \\
\hline 1998 & Júbilo Iwata & J1 League & 0 & 0 & 0 & 0 & 0 & 0 & 0 & 0 \\
\hline 2001 & \multirow{10}{*}{ Ventforet Kofu } & \multirow{5}{*}{ J2 League } & 35 & 4 & 3 & 0 & 2 & 0 & 40 & 4 \\
\hline 2002 & & & 33 & 5 & 2 & 0 & & & 35 & 5 \\
\hline 2003 & & & 39 & 9 & 1 & 0 & & & 40 & 9 \\
\hline 2004 & & & 28 & 2 & 1 & 0 & & & 29 & 2 \\
\hline 2005 & & & 41 & 10 & 2 & 0 & & & 43 & 10 \\
\hline 2006 & & \multirow{2}{*}{ J1 League } & 26 & 2 & 3 & 1 & 1 & 0 & 30 & 3 \\
\hline 2007 & & & 32 & 2 & 1 & 0 & 7 & 0 & 40 & 2 \\
\hline 2008 & & \multirow{3}{*}{ J2 League } & 38 & 3 & 1 & 0 & & & 39 & 3 \\
\hline 2009 & & & 50 & 2 & 2 & 0 & & & 52 & 2 \\
\hline 2010 & & & 32 & 2 & 1 & 0 & & & 33 & 2 \\
\hline Country & \multicolumn{2}{|c|}{ Japan } & 354 & 41 & 15 & 1 & 10 & 0 & 379 & 42 \\
\hline \multicolumn{3}{|c|}{ Total } & 354 & 41 & 15 & 1 & 10 & 0 & 379 & 42 \\
\hline
\end{tabular}

Target sentence: After 2 years blank, Ken Fujita joined the J2 League club Ventforet Kofu in 2001.

Figure 2: TOTTO example with complex table structure and temporal reasoning.

Table Title: Shuttle America

Section Title: Fleet

Table Description: As of January 2017, the Shuttle America fleet consisted of the following aircraft:

\begin{tabular}{|c|c|c|c|c|c|c|c|c|}
\hline \multirow{2}{*}{ Aircraft } & \multirow{2}{*}{ Total } & \multirow{2}{*}{ Orders } & \multicolumn{4}{|c|}{ Passengers } & \multirow{2}{*}{ Operated For } & \multirow{2}{*}{ Notes } \\
\hline & & & $\mathbf{F}$ & $\mathrm{Y+}$ & $\mathbf{Y}$ & & & \\
\hline \multirow{2}{*}{ Embraer E170 } & 5 & - & 6 & 16 & \multirow{2}{*}{48} & 70 & United Express & transferred to Republic Airline \\
\hline & 14 & - & 9 & 12 & & 69 & \multirow{2}{*}{ Delta Connection Delta Shuttle } & \multirow{2}{*}{2 planes on wet lease from Republic Airline } \\
\hline Embraer E175 & 16 & - & 12 & 12 & 52 & 76 & & \\
\hline Total & 35 & - & & & & & & \\
\hline
\end{tabular}

Target sentence: Shuttle America operated the E-170 and the larger E-175 aircraft for Delta Air Lines,.

Figure 3: TOTTO example with rare topics and complex table structure.

Table Title: Pune - Nagpur Humsafar Express

Section Title: Schedule

Table Description: None

\begin{tabular}{|l|l|l|l|l|l|l|l|}
\hline Train Number & Station Code & Departure Station & Departure Time & Departure Day & Arrival Station & Arrival Time & Arrival Day \\
\hline 11417 & PUNE & Pune Junction & $22: 00$ PM & Thu & Nagpur Junction & 13:30 PM & Fri \\
\hline 11418 & NGP & Nagpur Junction & $15: 00$ PM & Fri & Pune Junction & 08:05 AM & Sat \\
\hline
\end{tabular}

Target sentence: The 11417 Pune - Nagpur Humsafar Express runs between Pune Junction and Nagpur Junction.

Figure 4: TOTTO example with rare topic. 
Table Title: Montpellier

Section Title: Climate

Table Description: None

\begin{tabular}{|c|c|c|c|c|c|c|c|c|c|c|c|c|c|}
\hline \multicolumn{14}{|c|}{ Climate data for Montpellier (1981-2010 averages) } \\
\hline Month & Jan & Feb & Mar & Apr & May & Jun & Jul & Aug & Sep & Oct & Nov & Dec & Year \\
\hline Record high ${ }^{\circ} \mathrm{C}\left({ }^{\circ} \mathrm{F}\right)$ & $\begin{array}{l}21.2 \\
(70.2)\end{array}$ & \begin{tabular}{|l|}
22.5 \\
$(72.5)$
\end{tabular} & \begin{tabular}{|l|}
27.4 \\
$(81.3)$
\end{tabular} & $\begin{array}{l}30.4 \\
(86.7)\end{array}$ & $\begin{array}{l}35.1 \\
(95.2)\end{array}$ & \begin{tabular}{|l|}
37.2 \\
$(99.0)$
\end{tabular} & \begin{tabular}{|l|}
37.5 \\
$(99.5)$
\end{tabular} & \begin{tabular}{|l|}
36.8 \\
$(98.2)$
\end{tabular} & \begin{tabular}{|l|}
36.3 \\
$(97.3)$
\end{tabular} & $\begin{array}{l}31.8 \\
(89.2)\end{array}$ & $\begin{array}{l}27.1 \\
(80.8)\end{array}$ & $\begin{array}{l}22.0 \\
(71.6)\end{array}$ & \begin{tabular}{|l|}
37.5 \\
$(99.5)$
\end{tabular} \\
\hline Average high ${ }^{\circ} \mathrm{C}\left({ }^{\circ} \mathrm{F}\right)$ & $\begin{array}{l}11.6 \\
(52.9)\end{array}$ & $\begin{array}{l}12.8 \\
(55.0)\end{array}$ & $\begin{array}{l}15.9 \\
(60.6)\end{array}$ & $\begin{array}{l}18.2 \\
(64.8)\end{array}$ & $\begin{array}{l}22.0 \\
(71.6)\end{array}$ & $\begin{array}{l}26.4 \\
(79.5)\end{array}$ & \begin{tabular}{|l|}
29.3 \\
$(84.7)$
\end{tabular} & $\begin{array}{l}28.9 \\
(84.0)\end{array}$ & \begin{tabular}{|l|}
25.0 \\
$(77.0)$
\end{tabular} & $\begin{array}{l}20.5 \\
(68.9)\end{array}$ & $\begin{array}{l}15.3 \\
(59.5)\end{array}$ & $\begin{array}{l}12.2 \\
(54.0)\end{array}$ & \begin{tabular}{|l|}
19.9 \\
$(67.8)$
\end{tabular} \\
\hline Daily mean ${ }^{\circ} \mathrm{C}\left({ }^{\circ} \mathrm{F}\right)$ & $\begin{array}{l}7.2 \\
(45.0)\end{array}$ & $\begin{array}{l}8.1 \\
(46.6)\end{array}$ & $\begin{array}{l}10.9 \\
(51.6)\end{array}$ & $\begin{array}{l}13.5 \\
(56.3)\end{array}$ & $\begin{array}{l}17.3 \\
(63.1)\end{array}$ & \begin{tabular}{|l|}
21.2 \\
$(70.2)$
\end{tabular} & $\begin{array}{l}24.1 \\
(75.4)\end{array}$ & \begin{tabular}{|l|}
23.7 \\
$(74.7)$
\end{tabular} & \begin{tabular}{|l|}
20.0 \\
$(68.0)$
\end{tabular} & $\begin{array}{l}16.2 \\
(61.2)\end{array}$ & $\begin{array}{l}11.1 \\
(52.0)\end{array}$ & \begin{tabular}{|l|}
8.0 \\
$(46.4)$
\end{tabular} & \begin{tabular}{|l|}
15.1 \\
$(59.2)$
\end{tabular} \\
\hline Average low ${ }^{\circ} \mathrm{C}\left({ }^{\circ} \mathrm{F}\right)$ & $\begin{array}{l}2.8 \\
(37.0)\end{array}$ & \begin{tabular}{|l|}
3.3 \\
$(37.9)$
\end{tabular} & \begin{tabular}{|l|}
5.9 \\
$(42.6)$
\end{tabular} & $\begin{array}{l}8.7 \\
(47.7)\end{array}$ & $\begin{array}{l}12.5 \\
(54.5)\end{array}$ & \begin{tabular}{|l|}
16.0 \\
$(60.8)$
\end{tabular} & \begin{tabular}{|l|}
18.9 \\
$(66.0)$
\end{tabular} & \begin{tabular}{|l|}
18.5 \\
$(65.3)$
\end{tabular} & \begin{tabular}{|l|}
15.0 \\
$(59.0)$
\end{tabular} & \begin{tabular}{|l|}
11.9 \\
$(53.4)$
\end{tabular} & $\begin{array}{l}6.8 \\
(44.2)\end{array}$ & \begin{tabular}{|l|}
3.7 \\
$(38.7)$
\end{tabular} & \begin{tabular}{|l|}
10.4 \\
$(50.7)$
\end{tabular} \\
\hline Record low ${ }^{\circ} \mathrm{C}\left({ }^{\circ} \mathrm{F}\right)$ & $-15(5)$ & $\begin{array}{l}-17.8 \\
(0.0)\end{array}$ & $\begin{array}{l}-9.6 \\
(14.7)\end{array}$ & $\begin{array}{l}-1.7 \\
(28.9)\end{array}$ & $\begin{array}{l}0.6 \\
(33.1)\end{array}$ & $\begin{array}{l}5.4 \\
(41.7)\end{array}$ & \begin{tabular}{|l|}
8.4 \\
$(47.1)$
\end{tabular} & \begin{tabular}{|l|}
8.2 \\
$(46.8)$
\end{tabular} & \begin{tabular}{|l|}
3.8 \\
$(38.8)$
\end{tabular} & $\begin{array}{l}-0.7 \\
(30.7)\end{array}$ & $-5(23)$ & \begin{tabular}{|l}
-12.4 \\
$(9.7)$
\end{tabular} & $\begin{array}{l}-17.8 \\
(0.0)\end{array}$ \\
\hline $\begin{array}{l}\text { Average precipitation } \mathrm{mm} \\
\text { (inches) }\end{array}$ & $\begin{array}{l}55.6 \\
(2.19)\end{array}$ & $\begin{array}{l}51.8 \\
(2.04)\end{array}$ & $\begin{array}{l}34.3 \\
(1.35)\end{array}$ & $\begin{array}{l}55.5 \\
(2.19)\end{array}$ & $\begin{array}{l}42.7 \\
(1.68)\end{array}$ & $\begin{array}{l}27.8 \\
(1.09)\end{array}$ & \begin{tabular}{|l|}
16.4 \\
$(0.65)$
\end{tabular} & $\begin{array}{l}34.4 \\
(1.35)\end{array}$ & $\begin{array}{l}80.3 \\
(3.16)\end{array}$ & $\begin{array}{l}96.8 \\
(3.81)\end{array}$ & $\begin{array}{l}66.8 \\
(2.63)\end{array}$ & \begin{tabular}{|l|}
66.7 \\
$(2.63)$
\end{tabular} & \begin{tabular}{|l|}
629.1 \\
$(24.77)$
\end{tabular} \\
\hline $\begin{array}{l}\text { Average precipitation } \\
\text { days }\end{array}$ & 5.5 & 4.4 & 4.7 & 5.7 & 4.9 & 3.6 & 2.4 & 3.6 & 4.6 & 6.8 & 6.1 & 5.6 & 57.8 \\
\hline Average snowy days & 0.6 & 0.7 & 0.3 & 0.0 & 0.0 & 0.0 & 0.0 & 0.0 & 0.0 & 0.0 & 0.1 & 0.7 & 2.4 \\
\hline $\begin{array}{l}\text { Average relative humidity } \\
(\%)\end{array}$ & 75 & 73 & 68 & 68 & 70 & 66 & 63 & 66 & 72 & 77 & 75 & 76 & 70.8 \\
\hline $\begin{array}{l}\text { Mean monthly sunshine } \\
\text { hours }\end{array}$ & 142.9 & 168.1 & 220.9 & 227.0 & 263.9 & 312.4 & 339.7 & 298.0 & 241.5 & 168.6 & 148.8 & 136.5 & $2,668.2$ \\
\hline \multicolumn{14}{|l|}{ Source \#1: Météo France } \\
\hline Source \#2: Infoclimat.fr (hum & hidity ar & snowy & s, 196 & 1990) & & & & & & & & & \\
\hline
\end{tabular}

Target sentence: Extreme temperatures of Montpellier have ranged from $-17.8^{\circ} \mathrm{C}$ recorded in February and up to $37.5^{\circ} \mathrm{C}\left(99.5^{\circ} \mathrm{F}\right)$ in July.

Figure 5: TOTTO example with interesting reference language. 